\title{
influencia del árido sobre la resistencia de los hormigones
}

Revue des Matériaux de Construction, n. ${ }^{\circ}$ 630, marzo 1968, págs. $116-118$

El primer investigador que propuso una fórmula para calcular la resistencia probable del hormigón en función de sus componentes fue el francés R. Feret (1896). Por sus trabajos sobre morteros y hormigones, R. Feret debe considerarse como el fundador de la ciencia del hormigón. Su fórmula es:

$$
R_{\mathrm{c}}=k\left(\frac{c}{1-(s+g)}\right)^{2}
$$

en la cual: $R_{\mathrm{c}}$ es la resistencia a compresión del hormigón; $c, s$ y $g$, los volúmenes absolutos del cemento, de la arena y de la grava, y $k$ un coeficiente que depende de la calidad del cemento. $\mathrm{Si}$ el hormigón es compacto, $1-(s+g)$ representa el volumen ocupado por el agua y el cemento, $c+e$, y la fórmula se convierte en:

$$
R_{\mathrm{c}}=k\left(\frac{1}{1+e / c}\right)^{2} \text {. }
$$

En 1925, Bolomey propuso una nueva fórmula, en la cual los componentes del hormigón se consideran en peso. Para hormigones compactos, la fórmula es la siguiente:

$$
R_{\mathrm{c}}=k\left(\frac{c}{e}-0,50\right)
$$

J. Bolomey no estaba satisfecho con esta fórmula, y en una publicación posterior (1) llamó la atención sobre la influencia de los áridos, que no intervienen sólo por su cantidad, sino también por su composición granulométrica, que modifica la cantidad necesaria de agua de amasado, y por el límite superior del árido. Bolomey sacó sus conclusiones de un estudio sobre seis composiciones granulométricas realizadas según Fuller, en las cuales hizo variar el tamaño superior del árido. Los resultados obtenidos con hormigones de consistencia plástica y una dosificación de $300 \mathrm{~kg}$ de cemento por $\mathrm{m}^{3}$ de hormigón, se encuentran en la tabla I. 
TABLA I

\begin{tabular}{|c|c|c|c|c|c|c|}
\hline $\begin{array}{l}\text { Límites del árido } \\
(\mathrm{mm})\end{array}$ & $0 / 4$ & $0 / 8$ & $0 / 15$ & $0 / 30$ & $0 / 60$ & $0 / 120$ \\
\hline $\begin{array}{l}\text { Agua de amasado } \\
\qquad\left(\mathrm{kg} / \mathrm{m}^{3}\right)\end{array}$ & 253 & 214 & 202 & 165 & 157 & 136 \\
\hline $\begin{array}{l}\text { Peso volúmico } \\
\left(\mathrm{kg} / \mathrm{m}^{3}\right)\end{array}$ & 2.173 & 2.260 & 2.317 & 2.385 & 2.447 & 2.476 \\
\hline $\begin{array}{l}\text { Resistencia a } \\
\text { compresión a los } \\
28 \text { días } \\
\left(\mathrm{kg} / \mathrm{cm}^{2}\right)\end{array}$ & 88 & 108 & 131 & 201 & 205 & 262 \\
\hline
\end{tabular}

Con estos resultados, Bolomey llega a la conclusión de que el agua de amasado necesaria viene determinada por el tamaño superior del árido y ha tratado de calcular esta cantidad de agua teniendo en cuenta el módulo de finura de Abrams. Para este cálculo, Bolomey ha determinado experimentalmente una serie de coeficientes en función de la finura y de la naturaleza del árido (canto rodado o piedra triturada).

El autor de este artículo cree que la reducción en la cantidad de agua de amasado y el aumento de la resistencia no están motivados por el tamaño superior del árido, sino por el hecho de que, respetando la curva granulométrica de Fuller, a medida que crece el tamaño máximo del árido, su contenido en arena se reduce, y por este motivo la cantidad de agua de amasado decrece.

La situación es completamente diferente si se parte de un mortero dado y si se introduce en este mortero grava sin cambiar su composición inicial. Las investigaciones hechas en este sentido no han conducido a resultados concordantes en razón de los diferentes materiales empleados y por el hecho frecuente de que de un caso particular se sacan conclusiones generales.

Así, Gilkey (2) ha afirmado que, a consistencia constante, la introducción de grava en un mortero conduce a la disminución de su resistencia.

O. Graf (3) ha sostenido que la resistencia del hormigón depende, en primer lugar, de la resistencia de su mortero y que, introduciendo grava o piedra triturada en un mortero sin cambiar la relación agua/cemento, la resistencia permanece prácticamente constante. Como ejemplo, O. Graf ha presentado los siguientes resultados:

\section{Hormigones secos}

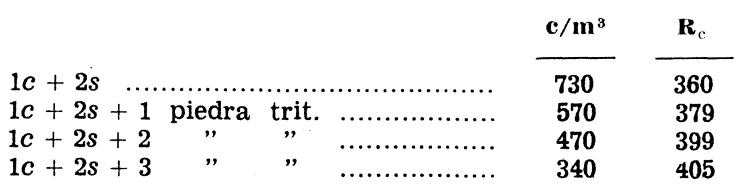

Hormigon s plásticos

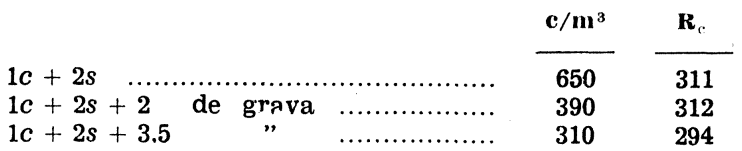


En la primera serie (hormigones secos), el aumento de la resistencia entre las mezclas extremas no es más que del $12 \%$, es decir, muy próximo al límite de errores experimentales $(+10 \%)$. En la segunda serie se observa una reducción de la resistencia del $5,5 \%$.

H. Burchartz (4), no estaba de acuerdo con la afirmación de Graf y demostró experimentalmente que, a consistencia constante, la introducción de árido grueso produce una disminución de la resistencia, que se acentúa más en el caso de mezclas plásticas.

La introducción de grava en un mortero puede producir los siguientes efectos:

a) La grava absorbe y adsorbe una parte del agua de amasado del mortero inicial, proporcionalmente a su porosidad y a la superficie total de sus granos, lo que tiene como consecuencia la reducción de la relación agua/cemento y debe provocar un aumento de la resistencia.

b) La presencia de grava en la masa del hormigón obtenido crea nuevos defectos, a saber:

- fisuras laminares entre el mortero y los granos de la grava, sobre todo cuando éstos son lisos;

- uniones más débiles entre el mortero y la grava que la resistencia a tracción de estos dos componentes (5);

- si se mantiene la consistencia constante, la introducción de grava en el mortero requiere un aumento de la relación agua/cemento, lo que provoca la disminución de la resistencia.

$\mathrm{Si}$, en lugar de grava, se introdujera en el mortero piedra triturada, la resistencia aumenta en razón de la superficie rugosa de sus áridos y de un mejor anclaje del mortero sobre esta superficie. Este hecho se ha demostrado experimentalmente (6) y (7), y explica también el aumento de resistencia confirmado por Graf en sus ensayos con hormigones secos, a base de piedra triturada.

Para estudiar la influencia predominante, al introducir grava en un mortero, el autor hizo hace tiempo las siguientes investigaciones (8):

Con una arena silícea de $0 / 5 \mathrm{~mm}$ y un cemento portland normal preparó tres morteros con dosificaciones en peso $1: 2,4 ; 1: 3,3 ; 1: 4,8$, a la misma consistencia, correspondiente a un escurrimiento de $48-51 \mathrm{~cm}$. En estos morteros se han introducido sucesivamente las fracciones de grava $5 / 15$ y $15 / 25 \mathrm{~mm}$, para llegar finalmente a tres hormigones de la misma consistencia, de la misma dosificación en peso $=1,6$ y con las composiciones granulométricas siguientes:

\begin{tabular}{cccc} 
Paso del tamiz & 5 & 15 & $25 \mathbf{~ m m}$ \\
\hline Curva I & 40 & 72 & 100 \\
Curva II & 55 & 82 & 100 \\
Curva III & 70 & 92 & 100
\end{tabular}

Los resultados obtenidos con estos hormigones de la misma consistencia se muestran en la tabla II. Se confirma que, para mantener la consistencia constante, la introducción de grava en el mortero requiere un aumento de la relación agua/cemento, lo que ha provocado una disminución de la resistencia. 
TABLA II

\begin{tabular}{|c|c|c|c|c|c|c|}
\hline \multirow{2}{*}{$\begin{array}{c}\text { Mortero } \\
\text { inicial }\end{array}$} & \multicolumn{3}{|c|}{ Composición del árido $(\mathrm{mm})$} & \multirow{2}{*}{$\mathbf{e} / \mathbf{c}$} & \multirow{2}{*}{$\begin{array}{l}\text { Escu- } \\
\text { rrimiento } \\
\quad(\mathrm{cm})\end{array}$} & \multirow{2}{*}{$\begin{array}{c}R_{\mathrm{c}}(28 \text { días }) \\
\left(\mathrm{kg} / \mathrm{cm}^{2}\right)\end{array}$} \\
\hline & $0 / 5$ & $5 / 15$ & $15 / 25$ & & & \\
\hline $1: 2,4$ & $\begin{array}{l}40 \\
40 \\
40\end{array}$ & $\begin{array}{l}- \\
32 \\
32\end{array}$ & - & $\begin{array}{l}0,50 \\
0,60 \\
0,66\end{array}$ & $\begin{array}{l}48 \\
46 \\
47\end{array}$ & $\begin{array}{l}448-\overline{27} \%) \\
326(-27 \%) \\
241(-46 \%)\end{array}$ \\
\hline $1: 3,3$ & $\begin{array}{l}55 \\
55 \\
55\end{array}$ & $\begin{array}{l}- \\
27 \\
27\end{array}$ & $\frac{-}{18}$ & $\begin{array}{l}0,64 \\
0,70 \\
0,70\end{array}$ & $\begin{array}{l}51 \\
49 \\
43\end{array}$ & $\begin{array}{l}289(-7 \%) \\
269(-7 \%) \\
235(-18 \%)\end{array}$ \\
\hline $1: 4,8$ & $\begin{array}{l}70 \\
70 \\
70\end{array}$ & $\begin{array}{l}- \\
22 \\
22\end{array}$ & $\frac{-}{8}$ & $\begin{array}{l}0,74 \\
0,79 \\
0,79\end{array}$ & $\begin{array}{l}50 \\
48 \\
44\end{array}$ & $\begin{array}{l}254(-\overline{20} \%) \\
202(-29 \%) \\
180(-29)\end{array}$ \\
\hline
\end{tabular}

Incluso, si se mantiene la relación agua/cemento constante, la introducción de grava en un mortero crea una disminución de la resistencia del hormigón obtenido, más pequeña que en el caso de la consistencia constante, como resulta de los datos experimentales mostrados en la tabla III.

TABLA III

\begin{tabular}{|c|c|c|c|c|c|c|}
\hline \multirow{2}{*}{$\begin{array}{c}\text { Mortero } \\
\text { inicial }\end{array}$} & \multicolumn{3}{|c|}{ Composición del árido $(\mathrm{mm})$} & \multirow{2}{*}{$\mathbf{e} / \mathbf{c}$} & \multirow{2}{*}{$\begin{array}{l}\text { Escu- } \\
\text { rrimiento } \\
(\mathrm{cm})\end{array}$} & \multirow{2}{*}{$\begin{array}{c}\mathbf{R}_{\mathrm{c}}(28 \text { días }) \\
\left(\mathrm{kg} / \mathrm{cm}^{2}\right)\end{array}$} \\
\hline & $0 / 5$ & $5 / 15$ & $15 / 25$ & & & \\
\hline \multirow{2}{*}{$1: 2,4$} & 40 & - & - & 0,50 & 47 & 448 \\
\hline & 40 & 32 & - & 0,50 & 32 & $400(-11 \%)$ \\
\hline $1: 3,3$ & $\begin{array}{l}55 \\
55 \\
55\end{array}$ & $\begin{array}{l}\overline{27} \\
27\end{array}$ & $\overline{\overline{-}}$ & $\begin{array}{l}0,64 \\
0,64 \\
0,64\end{array}$ & $\begin{array}{l}51 \\
41 \\
30\end{array}$ & $\begin{array}{l}289- \\
282(-2 \%) \\
255(-12 \%)\end{array}$ \\
\hline $1: 4,8$ & $\begin{array}{l}70 \\
70 \\
70\end{array}$ & $\begin{array}{l}\overline{22} \\
22\end{array}$ & $\overline{-}$ & $\begin{array}{l}0,74 \\
0,74 \\
0,74\end{array}$ & $\begin{array}{l}50 \\
39 \\
33\end{array}$ & $\begin{array}{l}254(-\overline{9} \%) \\
230(-24 \%) \\
192(-24)\end{array}$ \\
\hline
\end{tabular}

De estos resultados se puede concluir que, a relación agua/cemento constante, el introducir grava en un mortero crea una disminución de la resistencia en razón de los defectos estructurales provocados por la presencia de grava en la mezcla. La disminución de la resistencia es menor que en el caso de mezclas de la misma consistencia.

Casi en la misma época aparecieron dos estudios en los cuales se afirma que, a relación agua/cemento constante, la resistencia del hormigón a la compresión es independiente de la composición granulométrica del árido si la compactación se hace cuidadosamente, pero este compactado necesita un trabajo mecánico tanto más prolongado cuanto más arena contiene el árido (9) y (10).

En obra, es poco probable que pueda realizarse una compactación tan perfecta como en el laboratorio, sobre todo si las mezclas de hormigón son secas. En la práctica, se vibra el hormigón hasta que su cara superior esté bien cerrada y unida, pero esto no asegura que el hormigón se haya compactado en toda su masa. Esto se demostró en investigacio- 
nes realizadas por el autor en los hormigones de la presa de Bicaz (Rumania), durante los años 1950-52. Con las tres composiciones granulométricas siguientes

\begin{tabular}{|c|c|c|c|c|c|}
\hline Paso de tamiz & 3 & 7 & 15 & 30 & $60 \mathrm{~mm}$ \\
\hline Curva I & 23 & 35 & 50 & 70 & 100 \\
\hline Curva II & 32,5 & 50 & 70 & 100 & - \\
\hline Curva III & 50 & 70 & 100 & - & - \\
\hline
\end{tabular}

se prepararon hormigones con la misma dosificación en peso $1: 6,5$ y con la misma relación agua/cemento $=36$. Las probetas cúbicas vibradas dieron, después de 28 días de endurecimiento, los resultados reseñados en la tabla IV.

TABLA IV

\begin{tabular}{|c|c|c|c|}
\hline $\begin{array}{c}\text { Composición } \\
\text { granulométrica }\end{array}$ & $\mathbf{c} / \mathbf{m}^{3} \mathbf{( k g )}$ & $\begin{array}{c}\text { Peso volúmico } \\
\left(\mathbf{k g} / \mathbf{m}^{3}\right)\end{array}$ & $\mathbf{R}_{c}$ \\
\hline I & 319 & 2.525 & 415 \\
II & $316(-1 \%)$ & $2.425(-4 \%)$ & $364(-18 \%)$ \\
III & $298(-6 \%)$ & $2.360(-6 \%)$ & $226(-49 \%)$ \\
\hline
\end{tabular}

Se confirma que el peso volúmico y la resistencia a la compresión son tanto más reducidos cuanto más rico es el árido en fracciones finas, es decir, cuando la superficie total de los gránulos es más elevada. La disminución del peso volúmico del hormigón se debe en primer lugar al hinchamiento de la arena bajo el efecto de la humedad. Según las investigaciones del autor este hinchamiento se mantiene también cuando la arena húmeda se vibra con carga. La disminución de la resistencia a la compresión está motivada por este hinchamiento y por el hecho de que la misma cantidad de pasta de cemento debe dispersarse sobre una superficie de árido tanto mayor cuanto más rico es éste en arena, puesto que la relación pasta de conglomerante/superficie total de árido es más pequeña. B. G. Singh (11) ha llegado a la misma conclusión.

El primer autor que señaló la influencia de la relación arena/grava sobre las propiedades del hormigón fue Cantz (12). Esta influencia se manifiesta no sólo sobre las resistencias, sino también sobre todas las características de los hormigones. Para demostrarlo, el autor muestra en la tabla $\mathrm{V}$ los resultados de sus investigaciones realizadas con dos variedades de arena que se sustituyen progresivamente en la fracción 7/15 del árido. Los ensayos se realizaron sobre probetas prismáticas de $10 \times 10 \times 55 \mathrm{~cm}$. De estos resultados se deduce que aumentando la cantidad de arena en hormigones plásticos con la misma dosificación en peso $1: 6$, por el hecho de aumentar la superficie total del árido aumentan la relación agua/cemento y la retracción, en tanto que el peso volúmico, la dosificación real y la resistencia a la compresión disminuyen.

En los últimos años, otros investigadores han llegado a conclusiones parecidas a las que se exponen en este artículo.

St. Walker y D. L. Bloem han demostrado que hormigones de la misma dosificación y consistencia tienen una resistencia tanto más reducida cuanto más elevado es el límite superior del árido (13).

D. L. Bloem ha demostrado que con la misma relación agua/cemento y la misma dosificación, no es el hormigón con árido más grueso el de más alta resistencia (14). 
TABLA V

\begin{tabular}{|c|c|c|c|c|c|c|}
\hline \multirow{4}{*}{ 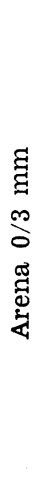 } & $\begin{array}{l}\text { Composición \% } \\
\text { del árido }\end{array}$ & $\begin{array}{c}0 / 3 \mathrm{~mm} \\
7 / 15 \mathrm{~mm} \\
15 / 30 \mathrm{~mm}\end{array}$ & $\begin{array}{l}30 \\
20 \\
50\end{array}$ & $\begin{array}{l}40 \\
10 \\
50\end{array}$ & $\frac{50}{50}$ & $\begin{array}{c}\text { Diferencia \% } \\
\text { entre los extremos }\end{array}$ \\
\hline & Hormigón fresco & $\begin{array}{c}e / c \\
\mathrm{c} / \mathrm{m}^{3} \\
\text { Peso vol. }\end{array}$ & $\begin{array}{c}0,55 \\
331 \\
2.502\end{array}$ & $\begin{array}{c}0,60 \\
321 \\
2.443\end{array}$ & $\begin{array}{c}0,67 \\
308 \\
2.369\end{array}$ & $\begin{array}{r}+21 \% \\
-\quad 7 \% \\
-\quad 5 \%\end{array}$ \\
\hline & $\begin{array}{l}\text { Resistencia a la } \\
\text { compresión } \\
\left(\mathrm{kg} / \mathrm{cm}^{2}\right)\end{array}$ & $\begin{array}{l}28 \text { días } \\
56 \text { días }\end{array}$ & $\begin{array}{l}179 \\
188\end{array}$ & $\begin{array}{l}132 \\
147\end{array}$ & $\begin{array}{l}124 \\
128\end{array}$ & $\begin{array}{l}-30 \% \\
-32 \%\end{array}$ \\
\hline & $\begin{array}{l}\text { Retracción } \\
\qquad(\mathrm{mm} / \mathrm{m})\end{array}$ & $\begin{array}{l}28 \text { días } \\
56 \text { días }\end{array}$ & $\begin{array}{l}0,16 \\
0,23\end{array}$ & $\begin{array}{l}0,22 \\
0.34\end{array}$ & $\begin{array}{l}0,25 \\
0,36\end{array}$ & $\begin{array}{l}+56 \% \\
+56 \%\end{array}$ \\
\hline \multirow{4}{*}{ 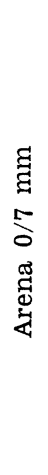 } & $\begin{array}{l}\text { Composición } \% \\
\text { del árido }\end{array}$ & $\begin{array}{c}0 / 7 \mathrm{~mm} \\
7 / 15 \mathrm{~mm} \\
15 / 30 \mathrm{~mm}\end{array}$ & $\begin{array}{l}30 \\
20 \\
50\end{array}$ & $\begin{array}{l}40 \\
10 \\
50\end{array}$ & $\frac{50}{50}$ & $\begin{array}{c}\text { Diferencia \% } \\
\text { entre los extremos }\end{array}$ \\
\hline & Hormigón fresco & $\begin{array}{c}e / c \\
c / \mathrm{m}^{3} \\
\text { Peso vol. }\end{array}$ & $\begin{array}{c}0,52 \\
329 \\
2.477\end{array}$ & $\begin{array}{c}0,59 \\
326 \\
2.474\end{array}$ & $\begin{array}{c}0,62 \\
317 \\
2.420\end{array}$ & $\begin{array}{r}+19 \% \\
-\quad 4 \% \\
-\quad 2 \%\end{array}$ \\
\hline & $\begin{array}{l}\text { Resistencia a la } \\
\text { compresión } \\
\left(\mathrm{kg} / \mathrm{cm}^{2}\right)\end{array}$ & $\begin{array}{l}28 \text { días } \\
56 \text { días }\end{array}$ & $\begin{array}{l}163 \\
203\end{array}$ & $\begin{array}{l}118 \\
159\end{array}$ & $\begin{array}{l}111 \\
147\end{array}$ & $\begin{array}{l}-31 \% \\
-27 \%\end{array}$ \\
\hline & $\begin{array}{l}\text { Retracción } \\
(\mathrm{mm} / \mathrm{m})\end{array}$ & $\begin{array}{l}28 \text { días } \\
56 \text { días }\end{array}$ & $\begin{array}{l}0,13 \\
0,25\end{array}$ & $\begin{array}{l}0,15 \\
0,30\end{array}$ & $\begin{array}{l}0,18 \\
0,31\end{array}$ & $\begin{array}{l}+38 \% \\
+24 \%\end{array}$ \\
\hline
\end{tabular}

D. L. Bloem y R. D. Gaynor han demostrado que la influencia del tamaño máximo del árido depende también de la dosificación del hormigón (15).

Finalmente, R. Zollinger ha demostrado que, para alcanzar altas resistencias a la compresión, la dosificación de cemento no debe determinarse por el volumen de los huecos del árido, sino por la superficie total de sus gránulos (16).

\section{CONCLUSIONES}

1) La arena, y sobre todo su fracción fina, ejerce una influencia mayor sobre las propiedades técnicas del hormigón que el cemento y la grava. Esta influencia se debe a la superficie total de los gránulos de la arena, que es muy grande en comparación con la del mismo peso de grava. Para una misma dosificación y una consistencia constante, el aumento de la superficie total del árido produce una reducción en la resistencia mecánica de los hormigones y acentúa la retracción, lo que conduce a una tendencia más pronunciada a la fisuración.

2) La introducción de grava en un mortero aumenta los defectos en la masa del hormigón obtenido, pero la disminución de las resistencias mecánicas es menor que la que se crea por la gran superficie total de la arena.

3) Todas las fórmulas propuestas hasta la fecha para determinar la posible resistencia del hormigón tienen un campo limitado de aplicación. Es necesario completarlas en 
el sentido propuesto por J. Bolomey (al menos para la fracción $0 / 15 \mathrm{~mm}$ del árido) con una característica que es función de la superficie total del árido y de la naturaleza de esta superficie: lisa o rugosa.

\section{BIBLIOGRAFIA}

1. Bolomey, J.: Module de finesse d'Abrams et calcul de l'eau de glâchage des bétons. Zürich, 1930.

2. Gilkey: ACI Journ., 1927.

3. Graf, O.: Aufbau des Mörtels und des Betons. Berlín 1930, págs. 3-4.

4. Burchartz, H.: Zement., 1929, I, pág. 2.

5. Alexander, K. M.: ACI Journ., noviembre 1959, 377. Valenta, O.: Conférence RILEM. Praga 1961, Rapport préliminaire, pág. 53.

6. Rothfuchs, G.: Betonstein-Ztg., septiembre 1959, 371.

7. Steopoe, A.: Rev. des Mat. de Constr., marzo 1961, 201.

8. Steopoe, A.: Bull. de 1Ecole Polytechn. Bucarest, 1941, 286.

9. Glanville, Collins y Matthews: Fef. Zement, 1938, 476.

10. Lenhard, H.: Zement, 1942, 123.

11. Singh, B. G.: ACI Journ., 1958, 897.

12. CANtz: Erfahrungen mit der Baukontrolle im Eissenbetonbau. Berlín, 1930. Pef. Graf, O.: Dtsch. Auss. für Eisenbeton. Cahier núm. 63, 1930, pág. 14.

13. Walker, St. y Bloem, L.: ACI Journ., septiembre 1960, 283.

14. Bloem, D. L.: Cement, Lime Gravel, 1961, junio, pág. 172.

15. Bloem, D. L. y Gaynor, R. D.: Proc. Am. Concr. Inst., 1960, 1429.

16. Zollinger, R.: Der Tiefbau, 1961, 114. 